\title{
Legacies of the Macquarie/AAO/Strasbourg H $\alpha$ Planetary Nebula Project (MASH): An International Workshop in Honour of the Career of Agnès Acker
}

\author{
Quentin A. Parker ${ }^{\mathrm{A}, \mathrm{B}, \mathrm{C}}$ \\ ${ }^{\text {A }}$ Macquarie University, Sydney, NSW 2109, Australia \\ B Anglo-Australian Observatory, Epping NSW 2121, Australia \\ ${ }^{\mathrm{C}}$ Email: qap@ics.mq.edu.au
}

Received 2009 July 17, accepted 2009 July 17

\begin{abstract}
A concise summary of the recent international workshop held in honour of the career of Agnès Acker is presented together with a brief exposition of her productive career in the study of Planetary Nebulae, the expelled, ionised outer shrouds of dying stars.
\end{abstract}

Keywords: stars: AGB and post-AGB — ISM: planetary nebulae: general — techniques: spectroscopic, surveys

\section{Workshop Summary}

A group of 42 astronomers met together at the ATNF lecture theatre in Epping, Sydney, Australia from 16 to 18 February 2009 to celebrate the career of Agnès Acker (www.astronomy.mq.edu.au/mash-workshop/).
This illustrious group included ten invited speakers: Agnès Acker, Robin Ciardullo, Romano Corradi, Martin Cohen, Orsola DeMarco, David Frew, George Jacoby, Warren Reid, Dick Shaw and Albert Zijlstra. Exactly half the attendees came from overseas from Spain, the

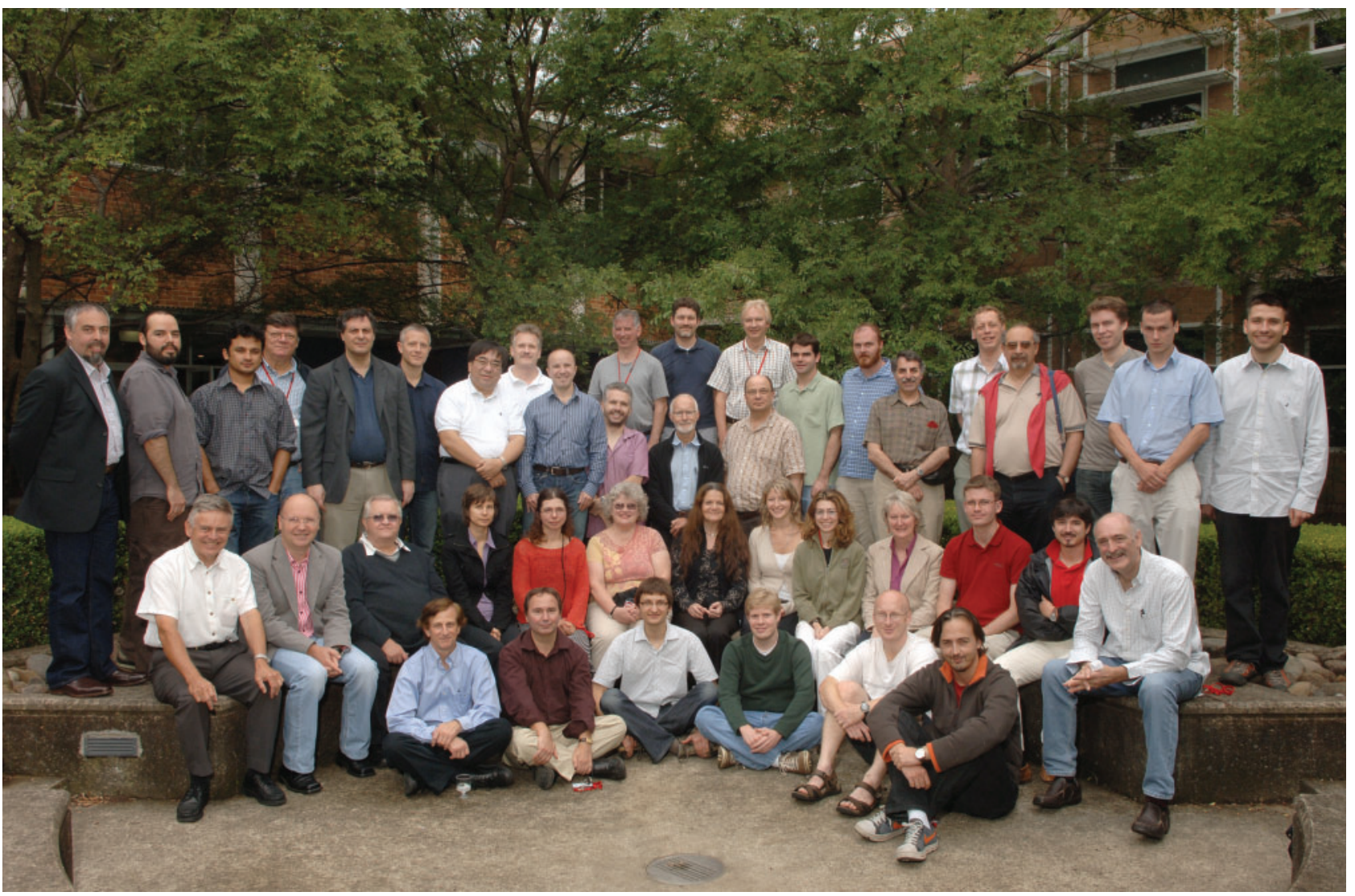

Figure 1 The workshop participants photograph taken in the internal courtyard of the ATNF outside the lecture theatre. 
UK, France, Germany, the United States of America and Hong Kong, with 21 participants coming from five universities/institutes within Australia.

The rest of the participants were: Richard Baxter (MQ), Ivan Bojicic (MQ), Russell Cannon (AAO), Rajan Chhetri (University of Sydney), Evan Crawford (UWS), Ain DeHorta (UWS), Kyle DePew (MQ), Paul Dobbie (AAO), Miroslav Filipovic (UWS), Ken Freeman (RSAA/ANU), David Frew (Perth), Corrado Giammanco (Instituto de Astrofsica de Canarias, Spain), Anne Green (University of Sydney), Martin Guerrero (Instituto de Astrofsica de Andaluca, Spain), Malcolm Hartley (AAO), Amanda Karakas (RSAA/ANU), Anna Kovacevic (Macquarie University), Maria Lugaro (Monash University), Julie Lutz (University of Washington, USA), Greg Madsen (University of Sydney), Luis Miranda (Instituto de Astrofsica de Andaluca, Spain), Brent Miszalski (MQ), Rodolfo Montez Jr (Rochester Institute of Technology, USA), Tara Murphy (University of Sydney), Simon O'Toole (AAO), Quentin Parker (MQ/AAO), Martin Roth (Astrophysikalisches Institut Potsdam, Germany), Laurence Sabin (Manchester University, UK), Christer Sandin (AIP, Germany), Milorad Stupar (AAO), Cezary Szyszka (ESO, Germany), Alan Vaughan (MQ), Chris Wareing (University of Leeds, UK) and Daniel Zucker (MQ).

The meeting was entitled 'Legacies of the Macquarie AAO Strasbourg H $\alpha$ Planetary Nebula project (MASH)' and had at its heart a celebration of planetary nebula (PN) discoveries and science resulting from the AAO/UKST H $\alpha$ survey (Parker et al. 2005). Senior scientists, mid-career researchers, postdoctoral fellows and $\mathrm{PhD}$ and masters students all came together in a convivial and friendly atmosphere to discuss the tremendous recent progress in unravelling the many complexities of the PN phenomena. This has been helped enormously by the significant new MASH discoveries and their subsequent and continuing exploitation (e.g. Parker et al. 2006; Miszalski et al. 2008).

The gathering comes just under 12 years since the international workshop 'Prospects for the AAO/UKST Galactic Plane H $\alpha$ Survey’ (e.g. Parker et al. 2008), which was motivated by the commencement of the underpinning wide-field survey that has led to so much new science and directly to the MASH discoveries, e.g. Parker et al. (2005). That meeting was also held in Sydney, 16-18 April 1997 and was also a refereed special PASA issue. This new meeting is a fitting testimony to the both this legacy of the $\mathrm{H} \alpha$ survey and the significant Australian role in PN research.

The workshop was split into several main topics over three days. On the first day, there was a session on PN discovery surveys in our own Galaxy and Magellanic clouds (introduced by invited reviews from Parker and Reid (e.g. Reid \& Parker 2010) and then one on PN observational techniques led by Frew, who described the progress in uncovering PN mimics via judicious use of plethora of multi-wavelength data now available (Frew \& Parker 2010, these proceedings). On the second day, there were sessions on the PN luminosity function introduced by a review by Ciardullo (2010; these proceedings and a special mention for being the first paper submitted) and more on observational techniques and general PN properties, including reviews by Corradi and Cohen. On the final day, there were sessions on $\mathrm{PNe}$ and their relation to late stage stellar evolution and the question of central star binarity that has been quite controversial over the last few years. There was also plenty of time set aside for discussions and debate concerning such questions as 'what is a Planetary Nebula?' and 'what is the role of binary stars in PNe formation?'. There was also as an interesting aside into the importance of the Hirsch index in astronomical careers moderated by George Jacoby for research fields, such as that of PN, that do not command the same glamour or attention as Cosmology or the hunt for extra-solar planets and dark energy.

Dick Shaw then gave an excellent conference summary that neatly encapsulated the key science discussed at the meeting. This included the tremendous increase in both the numbers and type of PN now available for study thanks to the MASH and other surveys; the current availability of new, multi-wavelength surveys that can now act as powerful complementary data sets with which to help unravel PN properties and eliminate the varied types of contaminants (such as Hir regions around hot stars, Symbiotic stars, CVs, and even SNRs and Pop-I Wolf-Rayet shells, see Frew \& Parker 2010) that have confounded previous compilations. Important concepts such as how do we recognise PNe and the importance of the new PN luminosity functions that are now taking shape for the well-defined local 2-3 Kpc volume, Galactic Bulge and Large Magellanic Cloud samples were also summarised. Finally, Shaw covered the exciting new results on the PN binary fraction uncovered (e.g. Miszalski et al. 2009) and the improvements in our understanding of abundances (Karakas 2010).

\section{A Brief Retrospective on the Career of Agnès Acker}

It is not everyday that one has the opportunity to honour a highly productive career that has spanned the golden age of a field of research. Fortunately we had this chance with this international workshop in honour of Agnès Acker. Agnès has been a leading light in the field of PNe research for nearly 40 years, producing over 260 publications and more than 3300 references. In the field of PNe research, this is a remarkable achievement.

Her work includes the seminal ESO/Strasbourg Catalogue of Galactic Planetary Nebulae (Acker et al. 1992, 1996). This is supplemented by major contributions to classification and understanding of Wolf-Rayet central stars of PNe, to spectrophotometry and chemical abundances of Galactic PNe and their evolution, ages and statistical properties. She continues her work with PNe with unabated enthusiasm to this day, despite being 'en retrait' since 2005. She has also made very strong contributions to the public face of Astronomy in France through her work with public planetaria as 'Directrice de la Publication de la 
Revue de 1APLF:Planetariums' (1995-current) and 'Fondatrice et presidente elue de 1 Association des Planetariums de Langue Francaise' since 1984. She has won many accolades and prizes over her highly productive career (http://astro.u-strasbg.fr/ acker/agnes. html).

On a personal note, Agnès has been a tremendous inspiration, a stalwart collaborator, a dear friend and a robust defender of what is decent and right. She has tremendous integrity, an indomitable spirit and a work ethic that defies belief, time, and, when on her bright yellow motorbike, sometimes gravity itself! Of particular merit is the care and concern she expresses towards her students, often knowing what is best for them when they sometimes do not know themselves. It has been a privilege to work closely with Agnès over the last nine years on the MASH project where her love of PNe and genuine interest in my own work and students has been greatly appreciated. I look forward to continued collaboration for many productive years to come.

\section{A Few Closing Remarks}

It has been a great pleasure and a tremendous personal privilege to help organise and host this meeting, full of such excellent scientists, like-minded colleagues and dear friends. The workshop size was ideal, which contributed to excited discussions, frank though friendly exchanges and stimulating presentations, all in a thoroughly unthreatening but professional environment. This was further aided by good coffee, excellent cakes and a decent lunch provided in the atrium outside the theatre. The conference dinner was held onboard a ship where we all enjoyed a spectacular Sydney Harbour cruise and even the poor weather that had kept us ensconced within the venue, let up sufficiently to permit a stroll around the deck without fear of falling overboard despite the open bar!
Several colleagues were unable to attend as originally intended (e.g. Stanghelini, Kastner, Dinerstein, Freeman) and were missed but not forgotten. Post-meeting feedback has been resoundingly positive and I hope you find the resulting refereed proceedings a fitting tribute to the vitality of this field of astrophysical research and a testimony to the quality and endeavour of all the contributors.

\section{Acknowledgments \\ I express thanks to the other members of the local organis- ing committee: Paul Dobbie, David Frew, Anna Kovacevic and Simon O'Toole for all their efforts in making the work- shop such a success. We thank the following organisations for financial and other support that made this workshop possible: Macquarie University, Macquarie Foundation for Astronomy, Anglo-Australian Observatory, Australia Telescope National Facility, University of Western Syd- ney, The ASA Donovan Trust and the Australian Academy of Science. I thank the Scientific Organising Committee for their construction of a broad and interesting pro- gramme (the SOC comprised: Quentin Parker (Chair), Agnès Acker, George Jacoby, Orsola De Marco, Sun Kwok and Dick Shaw). Finally I thank my co-editor David Frew for his valuable and knowledgeable input to the initial reviewing process for the submitted manuscripts.}

\section{References}

Acker, A. et al., 1992, Strasbourg/ESO Catalogue of Galactic Planetary Nebulae, Garching

Acker, A. et al., 1996, 1st supplement to the SECGPN, Garching

Miszalski, B., Parker, Q. A., Acker, A. et al., 2008, MNRAS, 384,525

Miszalski, B., Acker, A., Moffat, A., Parker, Q. A. \& Udalksi, A., A\&A, 496, 813

Parker, Q. A. \& Phillipps, S., 1998, PASA, 15, 28

Parker, Q. A., Phillipps, S., Pierce, M. J. et al., 2005, MNRAS, 362,689

Parker, Q. A., Acker, A., Frew, D. J. et al., 2006, MNRAS, 373, 79

Legacies of the Macquarie/AAO/Strasbourg $\mathrm{H} \alpha$ planetary nebula project
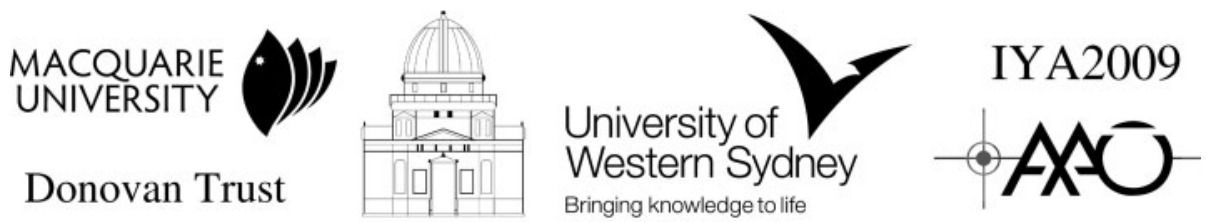

A conference in honour of Agnes Acker: 16-18th February 2009, Sydney, Australia 